\section{Preliminary analysis of partial reinforcement in discriminated avoidance}

\author{
D. GENE DAVENPORT, RICHARD D. OLSON*, and GAYLE A. OLSON† \\ St. Louis University, St. Louis. Mo. 63103
}

In Experiment 1 the effects of partial reinforcement $(0 \%, 25 \%, 50 \%, 75 \%$, or $100 \%)$ on acquisition and reinterpreted extinction of the lever response in discriminated avoidance was studied. In general, acquisition rate of the avoidance response was a direct function of reinforcement percentage, but with no evidence of a differential effect on extinction. In Experiment 2 an attempt to introduce partial reinforcement gradually under conditions of extended training demonstrated basically the same findings, with no increased resistance to extinction due to partial reinforcement, but found a dramatic "warm-up" for partial reinforcement. This suggested the need to begin extinction immediately following acquisition rather than at the beginning of the next session.

Special emphasis has recently been placed on the problem of the proper procedure for studying extinction of the instrumental response acquired in the discriminated-avoidance paradigm (Katzev, 1967; Davenport \& Olson, 1968). Davenport and Olson noted that a characteristic of the extinction procedure in reward training studies was not being represented in the extinction procedure for avoidance conditioning. As traditionally used in avoidance research, "extinction" involves omitting the shock whether or not the instrumental response occurs, thus making the response "unnecessary" for the omission of shock. In contrast, what characterizes reward training extinction is the fact that the response was no longer "effective" in producing the reward, which, in avoidance conditioning, would involve arranging contingencies so that the instrumental response could not prevent the occurrence of the shock. Thus, while the traditional procedure would extinguish the organism's fear and indirectly reduce the tendency to perform the response via a motivational change, the new procedure would leave fear relatively constant and extinguish the instrumental response perse. The reported findings were in agreement with this assumption-a rapid and consistent reduction in response tendency when the response was made ineffective in avoiding shock.

The consideration of partial reinforcement (PRF) is one of several research areas that is influenced by the reinterpretation of extinction in avoidance conditioning. PRF is an intermittent pairing of the cue and shock under the

*Experiment 1 is based on a the sis submitted by the second author to the Graduate School, St. Louis Lniversity, in partial fulfillment of requirements for the MS degree. Now at Louisiana State Liniversity of New Orleans. La. 70122.

$\div$ Now at Dominican College. New Orleans. La. traditional conception and, as such, has been used to explain the extended responding following removal of shock, such as reported by Solomon \& Wynne (1954), although these authors found little support for this possibility. Even if present, under the revised interpretation the intermittent pairing of the cue and shock would make the fear reaction to the warning signal more durable in the absence of shock, but is not comparable to the PRF effects of reward training. To be consistent with reward training, PRF involves the intermittent effectiveness of a response to produce "reinforcement," which in the avoidance case is the termination of the and 1 day of extinction $(N=5)$. warning signal and/or the omission of shock.

Specifically. this means, in the case of discriminated avoidance, that on "nonreinforced" trials the shock inevitably follows the warning signal, with the response ineffective in preventing the shock; and this situation is intermittently programmed with the alternative "reinforcement" trials where the shock is omitted, if the response occurs. Accordingly, the purpose of the present studies was to investigate the effects of PRF on the acquisition and resistance to extinction of a leverpress response in the discriminated-avoidance paradigm, using this revised interpretation of PRF and extinction.

\section{EXPERIMENT 1 Subjects}

Ss were 25 experimentally naive female Sprague-Dawley albino rats obtained from the colony at the St. Louis University Medical School. All Ss were between 110 and 130 days old when tested, weighed between 180 and $240 \mathrm{~g}$, and were maintained on ad lib food and water under reversed day-night lighting.

Apparatus

A standard single-lever Gerbrands operant-conditioning box was used in testing and was enclosed in a sound-suppressing chamber, with the appropriate programming and recording equipment housed in a separate room.

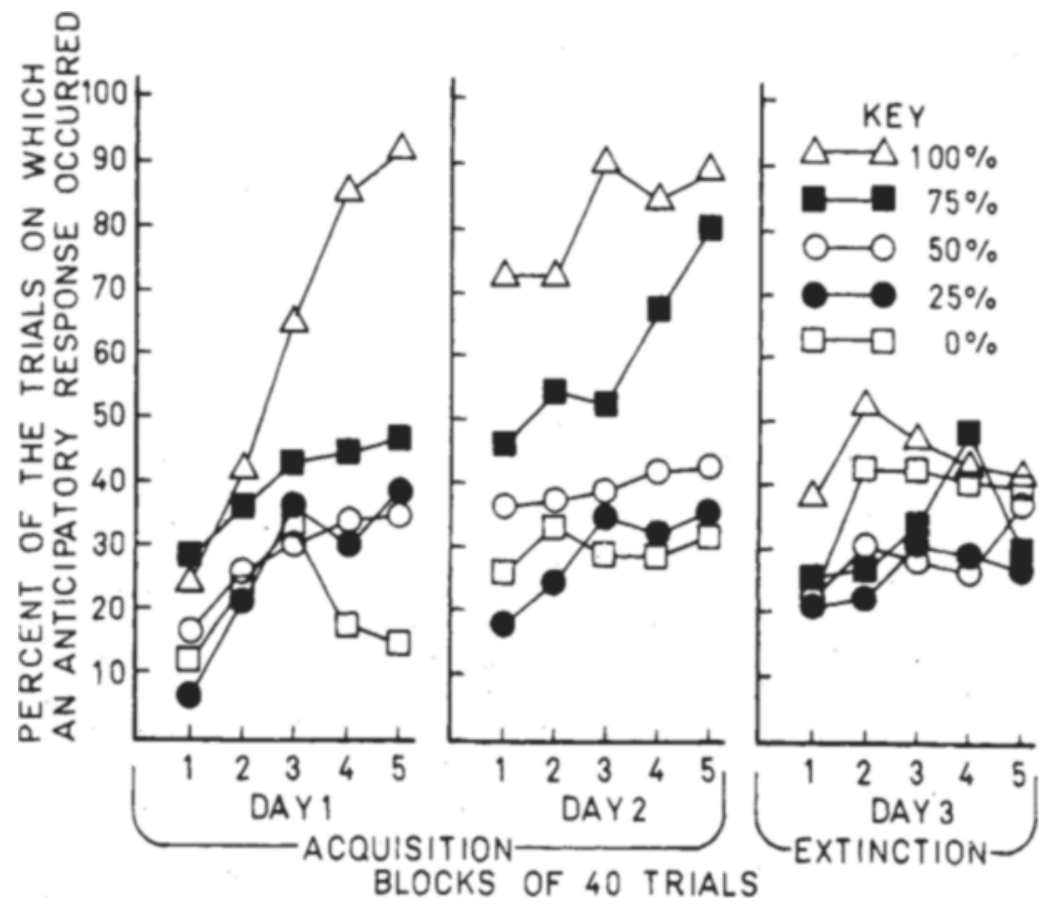

Fig. 1. Mean percent of the trials on which an anticipatory response occurred in blocks of 40 trials for $0 \%, 25 \%, 50 \%, 75 \%$, and $100 \%$ reinforced Ss during 2 days of acquisition 


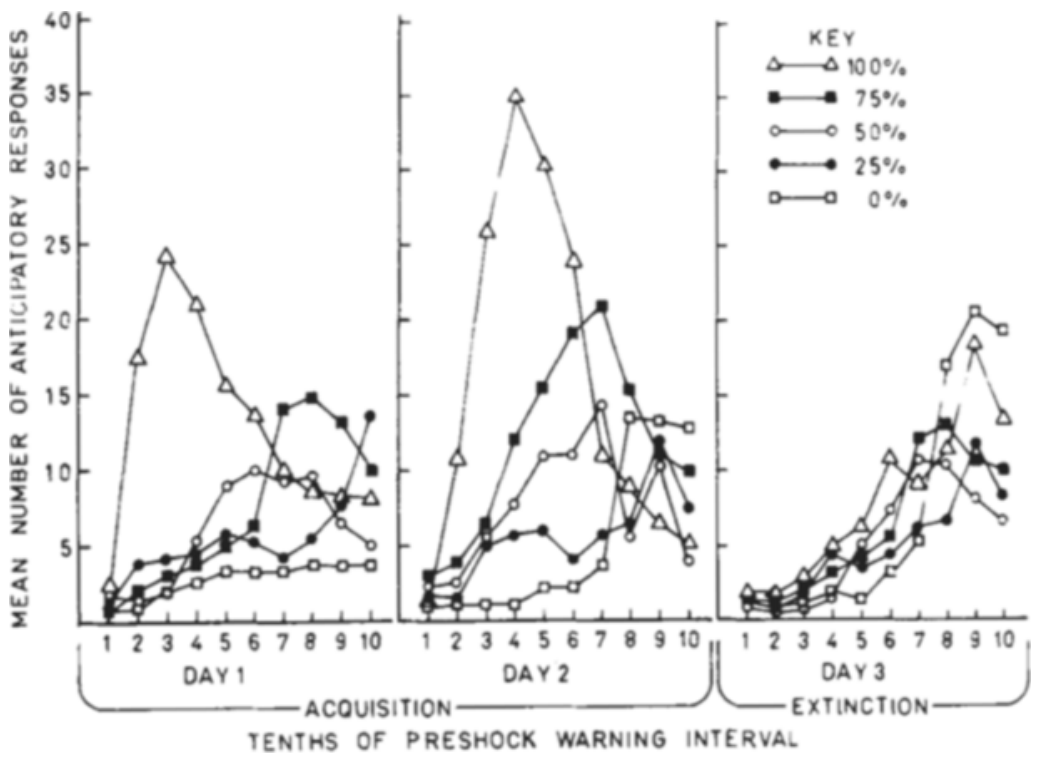

Fig. 2. Mean latency distributions for the $0 \%, 25 \%, 50 \%, 75 \%$, and $100 \%$ reinforced Ss in 10 ths of the warning interval for 2 days of acquisition and 1 day of extinction $(N=5)$.

Electric shock of .6-mA intensity was used as the aversive stimulus and was applied to the grid floor from a Lehigh Valley constant-current shocker with scrambler. Procedure

Five experimental groups of five Ss each $(0 \%, \quad 25 \%, 50 \%, 75 \%$, and $100 \%$ reinforcement) were given discriminated avoidance-escape training with a 10 -sec warning signal. A lever response during the light-clicker compound warning signal terminated the signal and prevented the shock from occurring when reinforcement was programmed, or, if not avoided, the same response terminated the shock and warning signal. If nonreinforcement was programmed, the shock followed the 10 -sec warning signal whether or not the response preceded the shock, and another leverpress was required to escape the shock. The study was run in five replications of five Ss each, with Ss randomly assigned to one of the five reinforcement conditions present in each replication. All Ss received 2 days of acquisition and 1 day of extinction (revised definition), 200 trials per day, with an average intertrial interval of $22.5 \mathrm{sec}$.

It is important to note that extinction was initiated at the beginning of the third day of training, a procedure that may have been inadvisable, as will be pointed out later.

\section{Results and Discussion}

Fig. 1 presents the mean percentage of the trials on which anticipatory response occurred, considered over blocks of 40 trials. An analysis of variance performed on the data for Day 1 showed that there was a significant main effect due to trials $(\mathrm{F}=110.2, \mathrm{p}<.01, \mathrm{df}=4 / 104)$, indicating an overall increase in responding over the five blocks of trials, and a significant Trials by Reinforcement Percentage interaction $(F=26.5, \quad p<.01, \quad d f=16 / 104)$, demonstrating that the rate of increase in responding was different for the various reinforcement percentages.

The same analysis applied to the Day 2 data now showed a significant main effect due to reinforcement percentage $(F=4.67$, $p<.01, d f=4 / 124)$ and that the groups were still increasing in response percentage $(F=304.8, p<.01, d f=4 / 104)$ and not all at the same rate $(F=163.96, p<.01$, $\mathrm{df}=16 / 104)$. Orthogonal comparisons on Day 2 showed Groups $100 \%$ and $75 \%$ made a significantly higher percentage of avoidance responses than the remaining groups $(F=16.8$ and 14.3 , respectively, $\mathrm{p}<.01, \quad \mathrm{df}=1 / 20)$. In general, the acquisition rate of the avoidance response was a direct function of reinforcement percentage, and, within the limits of the final levels of performance, although the $75 \%$ and $100 \%$ groups were not statistically different on the last block of 40 trials on Day 2.

These findings with respect to the acquisition effect of PRF are in general agreement with food reward studies when limited training is used (Jenkins \& Stanley, 1950; Lewis, 1960). However, the present study could demonstrate no increased resistance to extinction due to PRF. Neither the main effect of trials $(F<1)$ and reinforcement percentage $(F=2.17$, $p>.05, d f=4 / 120)$ nor the interaction of these two $(F<1)$ were significant. indicating that all extinction effects had occurred within the first block of 40 trials and that the rate of extinction was not influenced by reinforcement percentage acquisition trials used, reached different during acquisition. To confirm the finding of Davenport \& Olson (1968), a comparison of the $100 \%$ and $0 \%$ groups was made and showed a significant superiority during extinction trials for the $100 \%$ group. indicating some effects of conditioning $(F=4.79, \quad p<.05$, $\mathrm{df}=1 / 25)$.

Figure 2 presents the results of the five percentage-reinforcement groups in terms of a frequency distribution of the latencies of the anticipatory responses. There would appear to be a clear inverse relationship between modal latency and reinforcement percentage and, as reported by Davenport \& Olson (1968), there is a dramatic shift in peak frequency during extinction, as well as the general reduced tendency for the anticipatory response to occur.

\section{EXPERIMENT 2}

The possibility exists in Experiment 1 that there was not sufficient training. When the $100 \%$ and $75 \%$ groups were compared on the last block of 40 trials, they were quite comparable, although they were not when the performance over the complete 200 trials for Day 2 was included. In addition. food reward studies using the leverpress response frequently introduce the intermittent reinforcement schedule gradually, so that the lower reinforcement percentages occur only after extensive training at higher percentages.

The purpose of the second experiment was to test for increased resistances to extinction due to PRF after extended training and when the reinforcement percentages of the group were reduced gradually from $100 \%$. It was supposed that this might result in comparable asymptopic performance for the two groups and make the increased resistance to extinction for PRF more obvious.

Subjects and Apparatls

Three rats from the 100\% groups and three from the $75 \%$ group of Experiment 1 that had performed well during acquisition were placed in the continuous reinforcement (CRF) and partial reinforcement (PRF) groups, respectively. The apparatus was the same as that described in Experiment 1.

Procedure

The parameters of Experiment 1 were maintained, with the following exceptions. In the present study only 160 trials were given each day, and Ss were trained every other day for 16 training days. All parameters involving shock intensity, interstimulus interval, intertrial interval, and response contingencies were the same. The plan was to train the PRF group at each percent-reinforcement level until the avoidance performance approximated that of the CRF group. Thus, the PRF group received 2 days at $100 \%$ and were then 


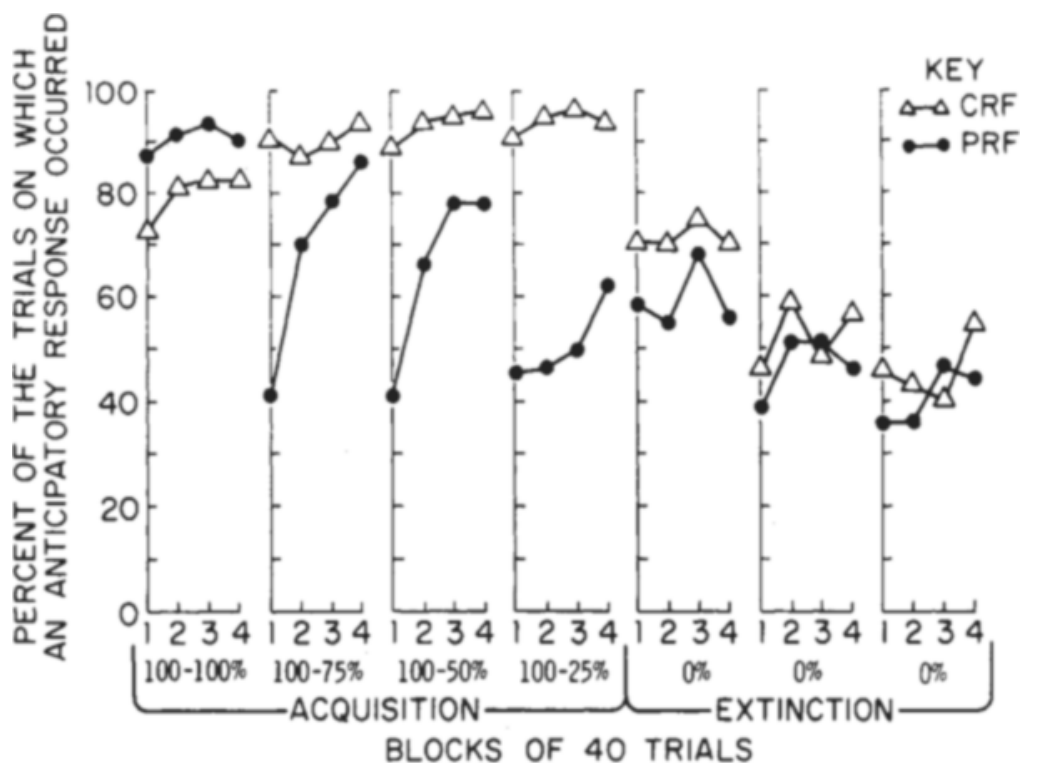

Fig. 3. Mean percent of the trials on which an anticipatory response occurred in blocks of 40 trials for the continuous reinforced (CRF) and partial reinforced (PRF) Ss on the last day of training at the percent shown, and for 3 days of extinction $(N=3)$.

maintained at $75 \%$ until Day 7 in one case and Day 11 in the other two cases. All three remained on $50 \%$ until Day 15 , when they were placed on $25 \%$ reinforcement for the last two training days. The original criterion of a performance equivalent to the CRF Ss before shifting was abandoned when it was apparent that stable performance was being reached, but not at the level of the CRF group. A further change was made based on the observation of a rather dramatic "warm-up" effect present in PRF Ss not present in the CRF group. This involved beginning extinction on the last part of Training Day 16, after "warm-up" was apparently complete.

\section{Results and Discussion}

The acquisition data for Experiment 2 is presented in Fig. 3, where values for the PRF group represent the Ss' performance at a given percentage reinforcement on the last day prior to the shift to the next lower level. The data for the CRF group are for the training day closest in time to that provided for PRF. An analysis of variance applied to the data verified the results of Experiment 1, with PRF group inferior to CRF $(F=38.9, p<.01, \mathrm{df}=1 / 22)$, in this case even with extended training and a gradually decreasing percentage. In Experiment 2, reinforcement percentage was confounded with amount of training for the PRF group and was a within-S variable. Nevertheless, the interaction of groups (CRF vs PRF) with "reinforcement percentage" ( $100 \%$ vs $100 \%$ to $75 \%$ to $50 \%$ to $25 \%)$ was significant $(\mathrm{F}=210.9$, the beginning of each day for the PRF, as compared to the CRF groups, suggested that the introduction of the extinction test on the next day after training in Experiment 1 , systematically opposed any increased resistance to extinction expected as a result of intermittent reinforcement. Therefore, as mentioned earlier, extinction was begun immediately following the acquisition session of 120 trials on Day 16, and with no warning. However, no partial reinforcement effect could be demonstrated even under these conditions. Actually, the CRF group produced reliably more responses than PRF $(F=16.42$, $\mathrm{p}<.01, \mathrm{df}=1 / 43$ ) and both declined in percentage of responses over blocks of 40 trials $(F=7.42, p<.01, \mathrm{df}=11 / 48)$, but with no difference in rate $(F<1.0)$. A close look at the extinction data of the first four blocks of extinction in Fig. 3 shows a much larger drop in responding from the last block of 40 trials of acquisition to the first block of 40 trials of extinction for the CRF group, with little change for the PRF group. However, no further change occurred during Day 1 extinction for either group. The problem of confounding warm-up with partial reinforcement effects on the remaining extinction sessions make these of uncertain value. The failure of the present study to obtain extinction effects seems more a function of a failure to obtain rapid extinction in the CRF group beyond that of the first block, rather than the lack of extended responding for PRF. Whether the increased responding in CRF is due to the extended training, the previous extinction provided in Experiment 1, or to peculiarities of beginning extinction immediately following training is not answerable from the present results. Research is currently evaluating this problem and other problems related to partial reinforcement effects in avoidance conditioning. decrease in reinforcemen little further change until an additional reinforcement percentage shift occurs.

However, a new systematic interaction was also reliably demonstrated and is clearly seen in Fig. 3 . At the beginning of each day's training, the performance for the PRF group was considerably inferior to that at the end of the session, suggesting a greater "warm-up" type of effect for the PRF group, with little or no "warm-up" for CRF $(F=36.31, p<.01$, df $=3 / 66)$. All three Ss in PRF showed this increase over blocks on each new day, and only one in the CRF group demonstrated even a slight "warm-up" effect. All main effects and their interactions were significant, indicating quite stable and dramatic effects of the variables studied, even with the small sample.

This reduced amount of responding at

\section{REFERENCES}

DAVENPORT, D. G., \& OLSON, R. D. A reinterpretation of extinction in discriminated avoidance. Psychonomic Science, 1968, 13, 5-6.

KATZEV, R. Extinguishing avoidance responses as a function of delayed warning signal termination. Journal of Experimental Psychology, 1967, 75, 339-344.

JENKINS, W. O., \& STANLEY, J. C., JR. Partial reinforcement: $A$ review and critique. Psychological Bulletin, 1950, 47, 193-234.

LEWIS, D. J. Partial reinforcement: A selective review of the literature since 1950. Psychological Bulletin, 1960, 57, 1-28.

SOLOMON, R. L.. \& WYNNE, L. C. Traumatic avoidance learning: The principles of anxiety conservation and partial irreversibility. Psychological Review, 1954, 61, 353-385. 